\section{ARDS - Nouveaux concepts en ventilation mécanique}

Martin R. Lessard MD FRCPC
Le syndrome de détresse respiratoire aigu (Acute Respiratory Distress Syndrome, ARDS) ${ }^{1-3}$ est associé encore aujourd'hui à une mortalité élevée de l'ordre de 50 à $70 \% .4,5$ Bien que l'insuffisance respiratoire ne soit pas la cause première de décès, ${ }^{4}$ la ventilation mécanique de ces patients demeure problématique, l'ARDS étant un facteur de risque important des complications barotraumatiques associées à la ventilation artificielle. ${ }^{6}$ La discussion qui suit traite de la prise en charge ventilatoire du patient atteint d'un ARDS en tenant compte des nouvelles connaissances de l'atteinte pulmonaire caractéristique de l'ARDS.

\section{Caractéristiques de l'atteinte pulmonaire}

L'ARDS est caractérisé par une lésion alvéolo-capillaire pulmonaire résultant en une perméabilité vasculaire pulmonaire augmentée. ${ }^{3,7,8}$ Cette lésion de la barrière alvéolo-capillaire cause un oedème interstitiel et alvéolaire pulmonaire de type lésionnel ou de haute perméabilité. Cette atteinte alvéolo-capillaire résulte soit d'une atteinte directe (e.g., aspiration) soit d'une atteinte indirecte (e.g., choc septique, traumatisme sévère, transfusions multiples). ${ }^{3,5}$ Bien que l'augmentation de perméabilité vasculaire pulmonaire semble atteindre le poumon de façon diffuse, on n'observe pas une distribution homogène de l'oedème alvéolaire. ${ }^{8} \mathrm{En}$ effet, bien que sur la radiographie simple du thorax les infiltrats alvéolaires apparaissent distribués de façon diffuse, les études tomodensitométriques ont montré que la distribution de ces infiltrats est très inhomogène. ${ }^{9-13}$ L'atteinte pulmonaire prédomine au niveau des zones pulmonaires dépendantes (c'est-à-dire dorsales chez le patient couché) avec préservation des zones non-dépendantes (ventrales chez le patient couché). ${ }^{14}$ Les poumons de patients atteint d'un ARDS se divisent selon un modèle de trois zones. ${ }^{12}$ La zone pulmonaire non dépendante reçoit toute la ventilation et est souvent hyperaérée par rapport au nombre d'unités alvéolaires qu'elle contient. Une deuxième zone, intermédiaire, est composée d'unités alvéolaires non ventilées mais qui peuvent être recrutées et rendues accessibles à la ventilation en modifiant les paramètres de ventilation mécanique. ${ }^{13}$ Finalement une troisième zone pulmonaire, la plus atteinte et habituellement la plus dépendante, est inac- cessible à la ventilation. ${ }^{11,14}$ Dans l'ARDS la zone pulmonaire aérée, et donc accessible à la ventilation, est très réduite et peut représenter aussi peu que $20-30 \%$ de la capacité pulmonaire normale. ${ }^{11,12,15} \mathrm{D}^{\prime}$ 'où le concept des poumons de bébé (baby lung) proposé par Gattinoni et al. ${ }^{12}$ Dans l'ARDS on observe aussi une diminution importante de la compliance statique pulmonaire. Cette faible compliance statique traduit principalement la diminution du volume pulmonaire aéré." Quant à l'élasticité pulmonaire, elle est mieux mesurée par la compliance spécifique, c'est à dire la compliance statique corrigée pour le volume pulmonaire accessible à la ventilation. ${ }^{16}$ La compliance spécifique est peu modifiée dans l'ARDS, ce qui indique que l'élasticité des zones pulmonaires ventilées est à peu près normale. ${ }^{11,15,17} \mathrm{Ces}$ zones pulmonaires reçoivent donc la quasi totalité de la ventilation et se comportent suivant des caractéristiques mécaniques normales. Cette amputation fonctionnelle du volume pulmonaire représente le changement pathologique majeur dans l'ARDS, expliquant que l'approche ventilatoire de ces patients doive être modifiée par rapport au patient avec des poumons sains.

\section{Dommages pulmonaires associés à la ventilation mécanique}

Il est bien reconnu que la ventilation mécanique est associée à des complications barotraumatiques (pneumothorax, pneumomédiastin, emphysème interstitiel). ${ }^{6.18}$ Cependant ces dernières années, plusieurs études animales ont démontré que la ventilation mécanique avec des volumes courants ou des pressions élevées pouvait aussi causer rapidement une altération de la perméabilité alvéolo-capillaire et un dommage alvéolaire diffus semblables à l'atteinte observé dans l'ARDS. ${ }^{19-23}$ De plus, le déterminant principal du dommage alvéolaire est la surdistension alvéolaire, donc le volume alvéolaire, et non les hautes pressions de ventilation. ${ }^{20,24}$ Certains suggèrent d'ailleurs que le terme volotraumatisme serait plus approprié que barotraumatisme pour décrire les dommages pulmonaires associés à

Département d'Anesthésie-Réanimation, Hôpital de l'EnfantJésus et Université Laval, Québec, Canada G1J 1 Z4. 
TABLEAU Principes de prise en charge ventilatoire du patient avec un ARDS

\begin{tabular}{|c|c|c|}
\hline Mode de ventilation & Volume contrôlé & Assisté-contrôlé, SIMV \\
\hline Volume courant & 5 a $7 \mathrm{ml} \cdot \mathrm{kg}^{-1}$ & $\begin{array}{l}\text { Doit être réduit si Pplat }>35 \mathrm{cmH}_{2} \mathrm{O} \text {, ou supérieur au point d'inflexion } \\
\text { supérieur de la courbe } \mathrm{P}-\mathrm{V}\end{array}$ \\
\hline PEEP & $5 \mathrm{à} 15 \mathrm{cmH}_{2} \mathrm{O}$ & Idéalement déterminé selon le point d'inflexion inférieur de la courbe P-V \\
\hline Fréquence respiratoire & $\leq 20-25 \min ^{-1}$ & Eviter l'auto-PEEP \\
\hline $\mathrm{FIO}_{2}$ & $\leq 0.60$ & $\begin{array}{l}\text { Augmenter pour } \mathrm{SaO}_{2} \geq 90 \% \text { (si PEEP } \geq \text { point d'inflexion inférieur ou } \geq 15 \\
\mathrm{cmH}_{2} \mathrm{O} \text { ) }\end{array}$ \\
\hline Ratio I:E & $\leq 1: 1$ & Eviter l'auto-PEEP \\
\hline Pression de plateau inspiratoire & $\leq 35 \mathrm{cmH}_{2} \mathrm{O}$ & Idéalement déterminé selon le point d'inflexion supérieur de la courbe $P-V$ \\
\hline
\end{tabular}

Pplat: pression de plateau inspiratoire; Courbe P-V: courbe pression-volume du système respiratoire

la ventilation. ${ }^{25}$ En ce qui concerne la ventilation de poumons normaux, la signification clinique de ces études n'est pas claire. Cependant, étant donné une capacité pulmonaire accessible à la ventilation très réduite, la ventilation à hauts volumes courants peut sûrement constituer un risque de dommage alvéolaire chez le patient avec un ARDS. Ces dommages associés à la ventilation mécanique vont compliquer et aggraver l'insuffisance respiratoire.

\section{Objectifs de ventilation dans l'ARDS (Tableau)}

Les objectifs de la ventilation mécanique du patient atteint d'un ARDS seront:

1 Assurer des échanges gazeux acceptables

2 Minimiser les risques de dommage pulmonaire associés à la ventilation mécanique en adaptant cette ventilation à la mécanique respiratoire de l'ARDS.

\section{Volume courant}

Des volumes courants de 10 à $15 \mathrm{ml} \cdot \mathrm{kg}^{-1}$ sont fréquemment utilisés en ventilation mécanique. ${ }^{26.27}$ Bien qu'il s'agisse du double d'un volume courant spontané normal, ces grands volumes courants ne sont probablement pas un risque important chez le patient avec des poumons sains ayant un capacité pulmonaire normale. Cependant le patient atteint d'un ARDS sévère ne dispose plus que de 20 à $30 \%$ de sa capacité pulmonaire normale pour recevoir toute la ventilation. ${ }^{11,12} \mathrm{Ceci}$ implique que chacune des unités alvéolaires ventilées devrait recevoir un volume de 6 à 8 fois son volume courant alvéolaire normal. Suivant le modèle des poumons de bébé de l'ARDS, ${ }^{12}$ ceci correspondrait à ventiler un enfant de $15 \mathrm{~kg}$ avec un volume courant de 600 à $800 \mathrm{ml}$. Chez l'animal, une surdistension alvéolaire de cet ordre provoque rapidement un dommage alvéolaire et un bris de la barrière alvéolo-capillaire associé à un oedème pulmonaire. ${ }^{19-23}$

Comme chez le patient normal, la courbe pressionvolume du système respiratoire d'un patient avec un ARDS est d'allure sigmoïdienne avec un aplatissement

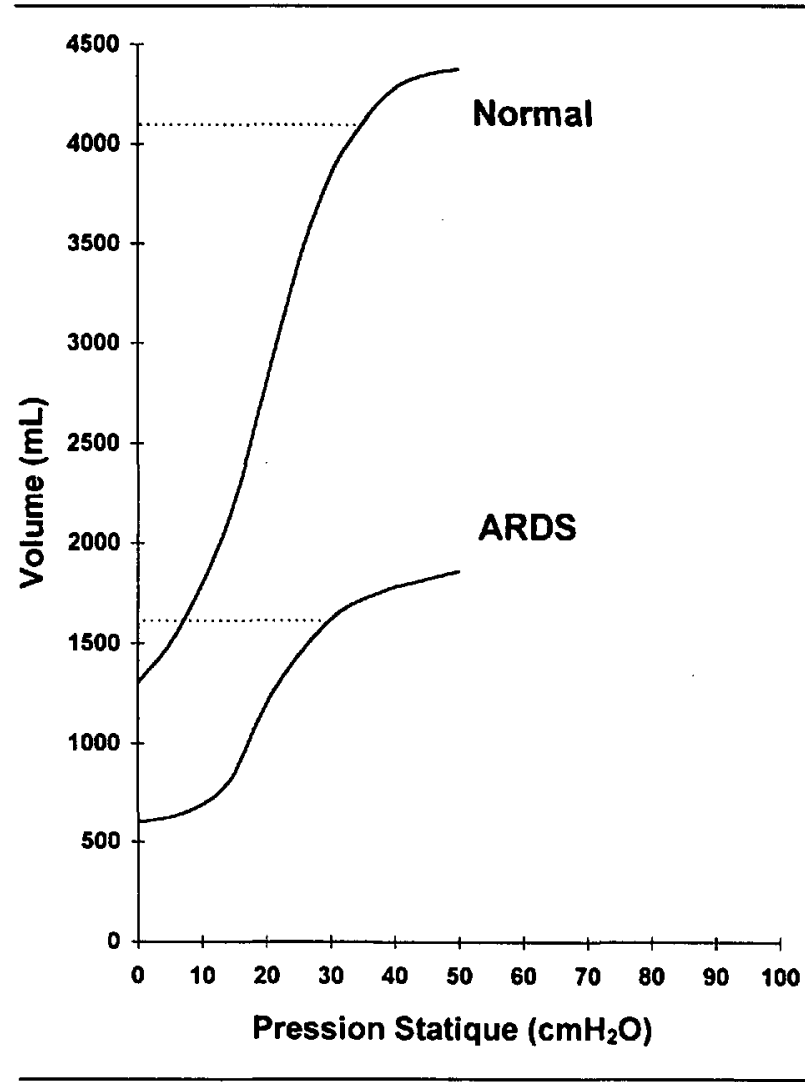

FIGURE 1 Courbe pression-volume du système respiratoire d'un patient sous ventilation mécanique sans pathologie pulmonaire (Normal) et d'un patient avec un ARDS. Les deux courbes ont une allure semblable mais avec une diminution de la capacité résiduelle fonctionnelle et un aplatissement supérieur (point d'inflexion supérieur) qui survient à un volume pulmonaire beaucoup plus faible chez le patient avec un ARDS. Ceci illustre l'amputation fonctionnelle du volume pulmonaire caractérisant l'ARDS.

supérieur indiquant l'atteinte de la capacité pulmonaire totale $^{28}$ (Figure 1). Cependant dans l'ARDS, cet aplatissement survient à un volume beaucoup plus faible, reflétant encore une fois l'amputation pulmonaire fonctionnelle de l'ARDS. ${ }^{28,29}$ Le point où débute cet 


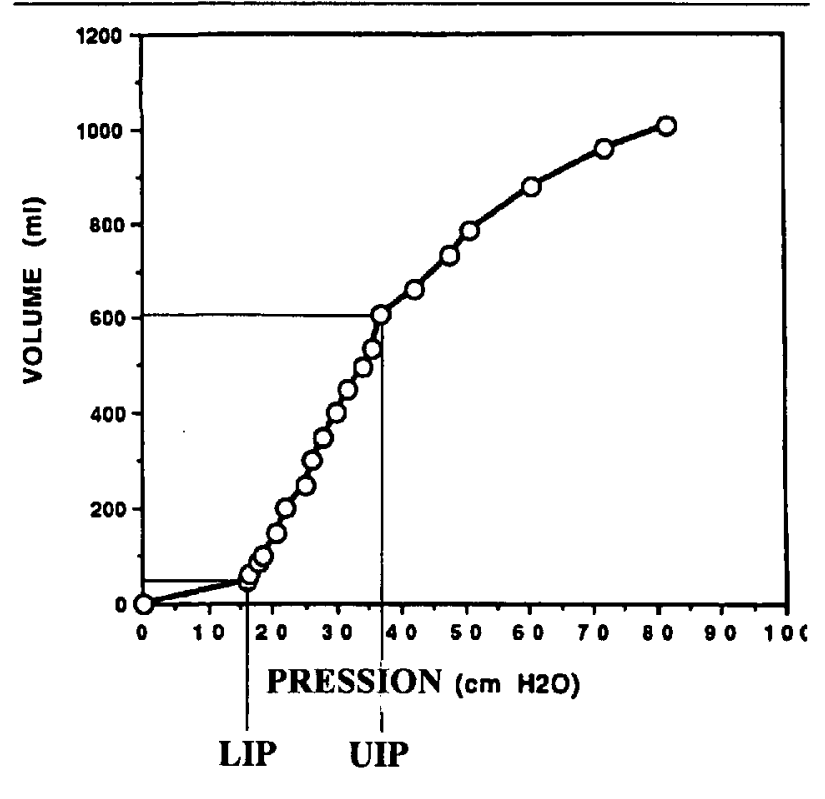

FIGURE 2 Courbe pression-volume d'un patient avec un ARDS. Les points d'inflexion inférieur et supérieur sont facilement identifiables. Le point d'inflexion supérieur (UIP) est à $36 \mathrm{cmH}_{2} \mathrm{O}$, correspondant à un volume de $600 \mathrm{ml}$ au-dessus de la capacité résiduelle fonctionnelle. Le point d'inflexion inférieur (LIP) correspond au point où la compliance du système respiratoire s'améliore brusquement ( 16 $\mathrm{cmH}_{2} \mathrm{O}$ ). (Courtoisie de Roupie E, et Brochard L, Créteil, France)

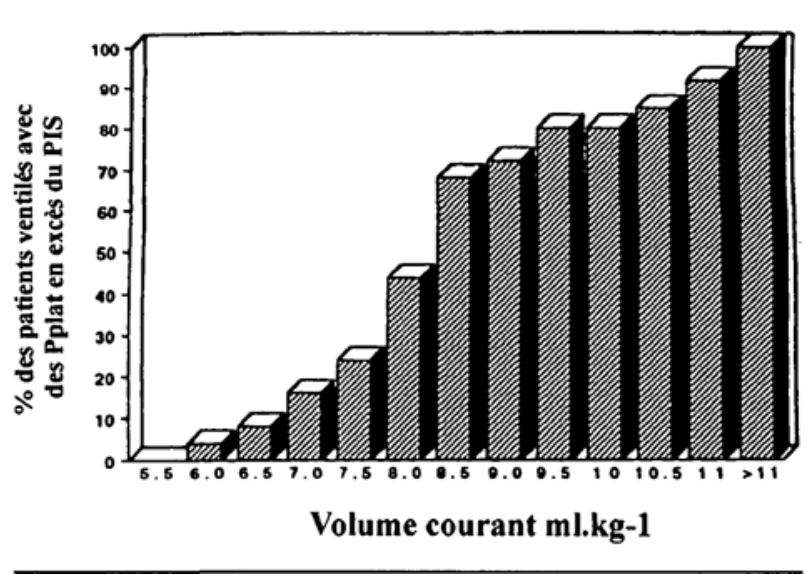

FIGURE 3 Proportion des patients avec un ARDS chez qui la pression de plateau inspiratoire excédait le point d'inflexion supérieur de la courbe pression-volume, en fonction du volume courant appliqué (avec un PEEP de $10 \pm 3 \mathrm{cmH}_{2} \mathrm{O}$ ). Avec un volume courant $\geq 10$ $\mathrm{ml} \cdot \mathrm{kg}^{-1}$, le point d'inflexion supérieur est dépassé chez $80 \%$ des patients. (Reproduit de Roupie E, et al. ${ }^{29}$ avec autorisation)

aplatissement est appelé point d'inflexion supérieur (Figure 2). Récemment Roupie et al. ont montré que la ventilation avec un volume courant de $10 \mathrm{ml} \cdot \mathrm{kg}$ dépasse la pression et le volume correspondant au point d'inflexion supérieur chez plus de $80 \%$ des patients avec ARDS (Figure 3). ${ }^{29}$ Ces volumes de ventilation exposent donc à une surdistension alvéolaire et aux baro-volotraumatismes associés. On recommande donc de limiter le volume courant à 5 à $7 \mathrm{ml} \cdot \mathrm{kg}^{-1}$ chez le patient avec ARDS. ${ }^{30-33}$ Idéalement, l'ajustement du volume courant devrait être guidé par la mesure de la courbe pression-volume du patient (Figure 2)..$^{29,34}$ Lorsque la mesure de la courbe pression-volume n'est pas possible, on peut plutôt monitorer la pression alvéolaire en mesurant la pression de plateau inspiratoire. Le volume courant doit être ajusté afin de garder la pression de plateau inspiratoire inférieure à $35 \mathrm{cmH}_{2} \mathrm{O} \cdot{ }^{31,35}$ Cependant cette limite est arbitraire, ne tient pas compte de la compliance pariétale, et excède fréquemment le point d'inflexion supérieur de la courbe pressionvolume. ${ }^{29}$ D'autres ont proposé d'analyser la courbe dynamique de pression de ventilation sur un enregistreur graphique ou sur l'écran du respirateur: si le débit inspiratoire est constant, une augmentation plus rapide de la pression de ventilation en fin d'inspiration (i.e. une concavité vers le haut de la courbe) suggère l'atteinte de la capacité alvéolaire maximale. ${ }^{36}$

\section{PEEP}

Il a été clairement démontré que l'utilisation prophylactique de PEEP ne permet pas de prévenir l'ARDS. ${ }^{37}$ $L$ 'ajout de PEEP permet de recruter un certain nombre d'alvéoles et de les maintenir ouvertes pendant tout le cycle respiratoire, améliorant ainsi l'oxygénation artérielle. Le PEEP augmente la capacité résiduelle fonctionnelle qui est très abaissée dans l'ARDS. ${ }^{15}$ En maintenant ouvertes les alvéoles ventilées, le PEEP, en plus d'améliorer l'oxygénation, diminue le cycle de fermeture et de ré-ouverture de ces conduits alvéolaires à chaque cycle de ventilation mécanique. Des études utilisant des modèles animaux d'ARDS ont montré que la tension nécessaire à la ré-ouverture répétée des petites voies aériennes aggrave le dommage pulmonaire. ${ }^{22.38}$ Ceci peut être diminué par l'application d'un niveau de PEEP permettant d'augmenter le volume pulmonaire en fin d'expiration et de maintenir ouvertes ces petites voies aériennes durant la totalité du cycle respiratoire. ${ }^{38}$ On peut donc reconnaître au PEEP deux effets bénéfiques dans l'ARDS: une amélioration de l'oxygénation artérielle, et une diminution du dommage pulmonaire associé à la ventilation. Cependant le PEEP a aussi des effets secondaires néfastes. La diminution du débit cardiaque est bien connue, et justifie parfois l'utilisation de monitoring hémodynamique invasif. De plus l'augmentation du volume pulmonaire produit par le PEEP peut aussi causer des dommages alvéolaires semblables à ceux provoqués par les grands volumes de ventilation. ${ }^{23}$ L'effet du PEEP n'est pas limité aux régions pul- 
monaires recrutables..$^{39}$ En même temps qu'il permet de recruter et maintenir ouvertes de nouvelles unités alvéolaires, le PEEP augmente aussi le volume de fin d'expiration des régions pulmonaires déjà bien ventilées. Cette augmentation de volume additionnée à l'effet du volume courant peut mener à une surdistension de certaines unités alvéolaires et les mettre à risque de dommage alvéolaire si le volume télé-inspiratoire (i.e., le volume courant additionné au volume induit par le PEEP) approche la capacité alvéolaire maximale (i.e., le point d'inflexion supérieur). ${ }^{23,29,40}$

Le niveau optimal de PEEP est fréquemment déterminé selon la mesure du transport d'oxygène. ${ }^{41}$ Cependant ceci peut conduire à l'application de niveaux très élevés de PEEP, ${ }^{42}$ exposant ainsi au risque de surdistension alvéolaire. La mesure de la courbe pressionvolume du système respiratoire peut aussi guider l'ajustement du PEEP (Figure 2). La portion inférieure de cette courbe est habituellement caractérisée par un changement de la pente de la courbe: le point d'inflexion inférieur. Ce point correspond au niveau de PEEP nécessaire pour un recrutement alvéolaire maximal. ${ }^{11,15,38,43,44} \mathrm{Ce}$ niveau de PEEP optimal est plutôt considéré comme le niveau minimum qui devrait être utilisé afin de prévenir la fermeture de petites voies aériennes en fin d'expiration. Au delà de ce niveau, l'augmentation du PEEP va principalement augmenter le volume des alvéoles déjà ventilées. ${ }^{11,15,44}$ L'ajustement du PEEP devra donc être guidé par plusieurs considérations: améliorer l'oxygénation artérielle et le transport d'oxygène, maintenir ouvertes un nombre maximal d'unités alvéolaires, et éviter la surdistension alvéolaire. Si on ne peut mesurer la courbe pression-volume, l'ajustement du PEEP se fait par augmentation progressive en surveillant les changements tant au niveau de l'oxygénation artérielle que de la pression de plateau inspiratoire. En pratique le niveau de PEEP optimal se situe habituellement entre 8 et $15 \mathrm{cmH}_{2} \mathrm{O}$ (parfois plus élevé si la compliance pariétale est abaissée). ${ }^{15,41}$ Il est important de rappeler que l'augmentation du PEEP peut exiger une diminution du volume courant afin de maintenir le volume téle-inspiratoire (le volume alvéolaire maximal) et la pression de plateau inspiratoire en dessous du point d'inflexion supérieur (ou $<35$ $\left.\mathrm{cmH}_{2} \mathrm{O}\right) .{ }^{23,29,40}$

\section{Fréquence respiratoire}

En ventilation mécanique conventionnelle, une fréquence respiratoire de 10 à 12 par minute est habituellement suffisante pour assurer une ventilation alvéolaire et une $\mathrm{PaCO}_{2}$ normales en raison des grands volumes courants utilisés. En réduisant les volumes courants on doit donc s'attendre à devoir augmenter la fréquence respiratoire. Il est toutefois difficile et risqué d'augmenter la fréquence au-delà de 20 à 25 par minute. En effet, ceci entraînerait une diminution excessive de la durée du cycle respiratoire et donc du temps expiratoire. Un temps expiratoire ne permettant pas une expiration complète va provoquer de l'hyperinflation pulmonaire avec auto-PEEP. ${ }^{45}$ Cet augmentation de volume pulmonaire télé-expiratoire va s'additionner à celle induite par le PEEP du respirateur, et résulter en une hyperinflation pulmonaire importante, augmentant ainsi les risques de surdistension alvéolaire.

\section{$\mathrm{FIO}_{2}$}

L'objectif est d'obtenir une oxygénation artérielle adéquate $\left(\mathrm{SaO}_{2} \geq 90 \%\right)$ avec une $\mathrm{FIO}_{2}$ considérée non toxique $(\leq 0.60) .{ }^{31}$ Dans les situations d'ARDS les plus sévères nécessitant une concentration d'oxygène élevé, on doit peser le risque entre, d'une part, celui associé à l'augmentation du PEEP et à la surdistension alvéolaire et, d'autre part, celui associé à l'administration d'une $\mathrm{FIO}_{2}$ au-delà des niveaux considérés non-toxiques. ${ }^{46} \mathrm{Il}$ est possible que dans cette situation, le risque de barovolotraumatisme l'emporte sur le risque de toxicité pulmonaire de l'oxygène.

\section{Ventilation en mode volume contrôlé ou en mode pression contrôlée}

La ventilation en mode volume contrôlé (VC) est associée à des pressions maximales parfois élevées. Dans le but de contrôler ces pressions et peut-être de réduire le risque de barotraumatisme, la ventilation en mode pression contrôlée (PC) a été proposée comme alternative. Effectivement lorsqu' on compare ces deux modes dans les mêmes conditions de ventilation (volume courant, fréquence, PEEP identiques), la pression maximale de ventilation est réduite en PC. Cependant aucune différence n'est observée au niveau de la pression de plateau inspiratoire. . $^{4748}$ Toute modification de la pression maximale de ventilation en manipulant la forme de débit inspiratoire ou en réduisant le débit inspiratoire sera sans effet sur le risque de dommage pulmonaire si elle n'est pas accompagnée d'une réduction de la pression alvéolaire (pression de plateau inspiratoire) par une diminution du volume courant ou du PEEP. ${ }^{23,49} \mathrm{Le}$ risque de dommage pulmonaire est associé au volume de distension alvéolaire, peu importe le mode de ventilation avec lequel ce volume est donné..$^{20,23}$ Un désavantage de la PC est l'absence de volume courant, et donc de ventilation minute, assurés, ce qui demande une surveillance beaucoup plus étroite tant des paramètres de ventilation que des échanges gazeux. ${ }^{50}$ Donc, bien que la PC puisse assurer adéquatement les échanges gazeux chez un patient atteint d'un ARDS, ${ }^{51}$ il n'existe actuelle- 
ment ni étude ni argument théorique suggérant que la PC soit supérieure à la VC. ${ }^{35,47,48}$

\section{Ventilation en ratio inversé}

La ventilation en ratio inversé (VRI) peut être utilisée en mode volume contrôlé (VC-VRI) ou mode pression contrôlée (PC-VRI). ${ }^{35}$ La prolongation du temps inspiratoire permet de réduire le débit inspiratoire et donc la pression maximale de ventilation. Cependant, tel que discuté dans le paragraphe précédent on ne doit pas en attendre une réduction du risque de baro-volotraumatisme si le volume pulmonaire et la pression de plateau inspiratoire demeurent inchangés. La prolongation du temps inspiratoire implique une réduction équivalente du temps expiratoire, et peut empêcher une expiration complète. Il en résulte une augmentation du volume pulmonaire télé-expiratoire avec auto-PEEP. ${ }^{35,47,50,52,53}$ Cet auto-PEEP augmente de façon très rapide lorsque le temps expiratoire diminue à moins de 1 seconde (i.e., un ratio I:E de 2:1 à une fréquence respiratoire de 20 par minute) ${ }^{35,50}$ L'auto-PEEP induit par la VRI s'ajoute au PEEP appliqué dans le circuit du respirateur, et de très hauts niveaux de pression alvéolaire télé-inspiratoire (PEEP total) et d'hyperinflation pulmonaire peut en résulter. Initialement des cas d'amélioration de l'oxygénation artérielle avec la VRI ont été rapportés. ${ }^{54,55}$ Cependant le niveau de PEEP total n'était pas mesuré ni pris en compte. ${ }^{56}$ Lorsque la VRI est comparée à la ventilation conventionnelle (VC) dans des conditions identiques de volume courant, fréquence respiratoire et PEEP total, aucune amélioration de l'oxygénation ne peut être observée. $47,52,53,57$ Au contraire l'augmentation de la pression alvéolaire moyenne associée à la VRI peut diminuer le retour veineux et le débit cardiaque, et donc le transport d'oxygène. ${ }^{47,52,58,59}$ L'utilisation de la VRI nécessite un monitoring respiratoire très étroit (volume courant, ventilation minute, PEEP total) et un monitoring hémodynamique invasif. En résumé, bien que ce mode soit suggéré par certains, il n'existe actuellement ni argument théorique suffisant ni d'étude contrôlée suggérant que la VRI soit supérieure à la VC conventionnelle et justifiant son utilisation dans l'ARDS.60

\section{Hypercapnie permissive}

Chez un patient atteint d'un ARDS sévère, la ventilation alvéolaire obtenue avec l'utilisation de volumes courants limités $\left(5-7 \mathrm{ml} \cdot \mathrm{kg}^{-1}\right)$ et d'une fréquence respiratoire qui doit aussi être limitée $\left(\leq 25 \mathrm{~min}^{-1}\right)$ sera fréquemment insuffisante pour rencontrer les besoins ventilatoires et maintenir une $\mathrm{PaCO}_{2}$ normale. On parle alors d'hypoventilation contrôlée et d'hypercapnie permissive. ${ }^{33,61,62}$ En général la $\mathrm{PaCO}_{2}$ s'élève aux environs de 60 à $80 \mathrm{mmHg}$, et le $\mathrm{pH}$ s'abaisse initialement autour de 7,25. Ce degré d'acidose est habituellement très bien toléré et ne nécessite aucune intervention..$^{29,33,61-63} \mathrm{Le}$ $\mathrm{pH}$ intracellulaire se corrige en quelques heures tandis que le $\mathrm{pH}$ extracellulaire retournera à des valeurs près de la normale en deux à trois jours par compensation métabolique normale. ${ }^{63}$ Dans certaines situations, une hypercapnie et une acidose beaucoup plus marquées peuvent survenir: $\mathrm{PaCO}_{2}>110 \mathrm{mmHg}$, $\mathrm{pH}<7,10 .{ }^{32}$ Certains tolèrent cette acidémie alors que d'autres suggèrent d'accélérer la compensation métabolique par une administration intraveineuse lente de bicarbonates de sodium. ${ }^{33,62}$ L'hypercapnie aiguë et chronique semble bien tolérée chez l'animal et l'humain si l'oxygénation est bien assurée. ${ }^{63}$ Plusieurs études ont montré que cette ventilation à bas volumes est bien tolérée tant au point de vue oxygénation artérielle que hémodynamique. ${ }^{64-66}$ Cependant une sédation profonde, et parfois la curarisation, sont nécessaires pour que le patient tolère cette hypercapnie. De plus certaines contre-indications doivent être respectées. Tout traumatisme crânien ou autre cause d'hypertension intracrânienne sont évidemment des contre-indications absolues à l'augmentation de la $\mathrm{PaCO}_{2}$. La stabilité cardiovasculaire est également une condition pour une bonne tolérance à l'acidose hypercapnique. Enfin la correction préalable d'une acidose métabolique associée est souhaitable afin d'éviter une acidémie trop sévère. Des études rétrospectives et prospectives suggèrent une réduction de la mortalité de l'ARDS, avec la ventilation limitée et l'hypercapnie permissive. ${ }^{32,61}$ Des études prospectives et randomisées sont en cours afin de confirmer ces résultats encourageants.

\section{Conclusion}

Au point de vue de la physiologie respiratoire, l'ARDS se caractérise par une amputation fonctionnelle du volume pulmonaire. Dans les cas sévères aussi peu que 20 à $30 \%$ des unités alvéolaires demeurent accessibles à la ventilation. La ventilation conventionnelle à hauts volumes risque donc de causer des dommages supplémentaires au poumon atteint d'un ARDS. L'approche ventilatoire doit être modifiée et adaptée aux caractéristiques physiologiques pulmonaires de l'ARDS. Un niveau de PEEP suffisant pour recruter un maximum d'alvéoles doit être appliqué mais en évitant la surdistension alvéolaire associée aux hauts niveaux du PEEP. Le volume courant doit aussi être réduit $\left(5-7 \mathrm{ml} \cdot \mathrm{kg}^{-1}\right)$ afin de limiter le volume alvéolaire téléinspiratoire et réduire les risques de dommage pulmonaire. Ce type de ventilation sera fréquemment insuffisant pour rencontrer la demande respiratoire du patient. On appelle donc cette stratégie ventilatoire hypercapnie permissive. Finalement, il n'existe actuellement ni argument théorique ni 
étude contrôlée suggérant que la ventilation en mode pression contrôlée ou en mode ratio inversé soit préférable à la ventilation en mode volume contrôlé.

"The skillful management of ARDS is a complex undertaking that requires mastery of the fundamental principles of lung protection and gas exchange efficiency ... As always, the best approach to such problems is a well-reasoned strategy firmly grounded in applied physiology." (From Marini JJ. ${ }^{67}$ )

\section{Références}

1 Ashbaugh DG, Bigelow DB, Petty TL, Levine BE. Acute respiratory distress in adults. Lancet 1967; 2: 319-23.

2 Murray JF, Matthay MA, Luce JM, Flick MR. An expanded definition of the adult respiratory distress syndrome. Am Rev Respir Dis 1988; 138: 720-3.

3 Bernard GR, Artigas A, Brigham KL, et al. The American-European Consensus Conference on ARDS. Definitions, mechanisms, relevant outcomes, and clinical trial coordination. Am J Respir Crit Care Med 1994; 149: 818-24.

4 Montgomery AB, Stager MA, Carrico CJ, Hudson LD. Causes of mortality in patients with the adult respiratory distress syndrome. Am Rev Respir Dis 1985; 132: 485-9.

5 Hudson LD, Milberg JA, Anardi D, Maunder RJ. Clinical risks for development of the acute respiratory distress syndrome. Am J Respir Crit Care Med 1995; 151: 293-301.

6 Gammon RB, Shin MS, Groves RH Jr, Hardin JM, Hsu C, $B u c h a l t e r S E$. Clinical risk factors for pulmonary barotrauma : a multivariate analysis. Am J Respir Crit Care Med 1995; 152: 1235-40.

7 Demling $R H$. Adult respiratory distress syndrome: current concepts. New Horizons 1993; 1: 388-401.

8 Sandiford P, Province MA, Schuster DP. Distribution of regional density and vascular permeability in the adult respiratory distress syndrome. Am J Respir Crit Care Med 1995; 151: 737-42.

9 Gattinoni L, Presenti A, Torresin A, et al. Adult respiratory distress syndrome profiles by computed tomography. J Thorac Imag 1986; 1: 25-30.

10 Maunder RJ, Shuman WP, McHugh JW, Marglin SI, Butler J. Preservation of normal lung regions in the adult respiratory distress syndrome: analysis by computed tomography. JAMA 1986; 255: 2463-5.

11 Gattinoni L, Pesenti A, Avalli L, Rossi F, Bombino M. Pressure-volume curve of total respiratory system in acute respiratory failure: computed tomographic scan study. Am Rev Respir Dis 1987; 136: 730-6.

12 Gattinoni L, Pesenti A. ARDS: the non-homogeneous lung; facts and hypothesis. Intensive Crit Care Digest 1987; 6: 1-4.

13 Gattinoni L, Pesenti A, Bombino M, et al. Relationships between lung computed tomographic density, gas exchange, and PEEP in acute respiratory failure. Anesthesiology 1988; 69: 824-32.

14 Pelosi P, D'Andrea L, Vitale G, Pesenti A, Gattinoni L. Vertical gradient of regional lung inflation in adult respiratory distress syndrome. Am J Respir Crit Care Med 1994; 149: 8-13.

15 Benito $S$, Lemaire $F$. Pulmonary pressure-volume relationship in acute respiratory distress syndrome in adults: role of positive end expiratory pressure. J Crit Care 1990; 5: 27-34.

16 Mancebo J, Benito S. Pulmonary mechanics in acute respiratory failure. Intensive Care World 1993; 10: 64-7.

17 Gattinoni L, D'Andrea L, Pelosi P, Vitale G, Pesenti A, Fumagalli $R$. Regional effects and mechanism of positive end-expiratory pressure in early adult respiratory distress syndrome. JAMA 1993; 269: 2122-7.

18 Gammon RB, Shin MS, Buchalter SE. Pulmonary barotrauma in mechanical ventilation: patterns and risk factors. Chest 1992; 102: 568-72.

19 Dreyfuss $D$, Basset $G$, Soler $P$, Saumon $G$. Intermittent positive-pressure hyperventilation with high inflation pressures produces pulmonary microvascular injury in rats. Am Rev Respir Dis 1985; 132: 880-4.

20 Dreyfuss $D$, Soler $P$, Basset $G$, Saumon $G$. High inflation pressure pulmonary edema: respective effects of high airway pressure, high tidal volume, and positive end-expiratory pressure. Am Rev Respir Dis 1988; 137: 1159-64.

21 Kolobow T, Moretti MP, Fumagalli $R$, et al. Severe impairment in lung function induced by high peak airway pressure during mechanical ventilation: an experimental study. Am Rev Respir Dis 1987; 135: 312-5.

22 Corbridge TC, Wood LDH, Crawford GP, Chudoba MJ, Yanos J, Sznajder JI. Adverse effects of large tidal volume and low PEEP in canine acid aspiration. Am Rev Respir Dis 1990; 142: 311-5.

23 Dreyfuss $D$, Saumon $G$. Role of tidal volume, FRC, and end-inspiratory volume in the development of pulmonary edema following mechanical ventilation. Am Rev Respir Dis 1993; 148: 1194-203.

24 Hernandez LA, Peevy KJ, Moise AA, Parker JC. Chest wall restriction limits high airway pressure-induced lung injury in young rabbits. J Appl Physiol 1989; 66: 2364-8.

25 Dreyfuss $D$, Soler $P$, Saumon $G$. Spontaneous resolution of pulmonary edema caused by short periods of cyclic overinflation. J Appl Physiol 1992; 72: 2081-9.

26 Snyder JV, Froese A. Respirator lung. In: Snyder JV, Pinsky MR (Eds.). Oxygen Transport in the Critically Ill. Chicago: Year Book Medical Publishers Inc., 1987: 358-73.

27 Shapiro BA, Peruzzi WT. Respiratory care. In: Miller RD (Ed.). Anesthesia, 4th ed. New York: Churchill Livingstone Inc., 1994: 2397-439.

28 Suter PM, Fairley $H B$, Isenberg $M D$. Effect of tidal vol- 
ume and positive end-expiratory pressure on compliance during mechanical ventilation. Chest 1978; 73: 158-62.

29 Roupie E, Dambrosio M, Servillo $G$, et al. Titration of tidal volume and induced hypercapnia in acute respiratory distress syndrome. Am J Respir Crit Care Med 1995; 152: 121-8.

$30 \mathrm{Hall} J B$, Wood $L D H$. Management of the patient on a ventilator. In: Hall JB, Schmidt GA, Wood LDH (Eds.). Principles of Critical Care. New York: McGraw-Hill Inc., 1992: 1617-33.

31 Slutsky AS. Mechanical Ventilation. ACCP consensus conference. Chest 1993; 104: 1833-59.

32 Hickling KG, Walsh J, Henderson S, Jackson R. Low mortality rate in adult respiratory distress syndrome using low-volume, pressure-limited ventilation with permissive hypercapnia: a prospective study. Crit Care Med 1994; 22: 1568-78.

33 Tuxen $D V$. Permissive hypercapnic ventilation. Am $\mathbf{J}$ Respir Crit Care Med 1994; 150: 870-4.

34 Levy P, Similowski $T$, Corbeil C, et al. A method for studying the static volume-pressure curves of the respiratory system during mechanical ventilation. J Crit Care 1989; 4: 83-9.

35 Marcy TW, Marini JJ. Inverse ratio ventilation in ARDS: rationale and implementation. Chest 1991; 100: 494-504.

36 Ranieri VM, Giuliani R, Fiore T, Dambrosio M, MilicEmili $J$. Volume-pressure curve of the respiratory system predicts effects of PEEP in ARDS: "occlusion" versus "constant flow" technique. Am J Respir Crit Care Med 1994; 149: 19-27.

37 Pepe PE, Hudson LD, Carrico CJ. Early application of positive end-expiratory pressure in patients at risk for the adult respiratory-distress syndrome. N Engl J Med 1984; 311: $281-6$.

38 Muscedere JG, Mullen JBM, Gan K, Slutsky AS. Tidal ventilation at low airway pressures can augment lung injury. Am J Respir Crit Care Med 1994; 149: 1327-34.

39 Gattinoni L, Pelosi P, Crotti S, Valenza F. Effects of positive end-expiratory pressure on regional distribution of tidal volume and recruitment in adult respiratory distress syndrome. Am J Respir Crit Care Med 1995; 151 : 1807-14.

40 Dreyfuss $D$, Saumon $G$. Should the lung be rested or recruited? The Charybdis and Scylla of ventilator management (Editorial). Am J Respir Crit Care Med 1994; 149: 1066-8.

41 Suter PM, Fairley HB, Isenberg $M D$. Optimum end-expiratory airway pressure in patients with acute pulmonary failure.'N Engl J Med 1975; 292: 284-9.

42 Kirby RR, Downs JB, Civetta JM, et al. High level positive end-expiratory pressure (PEEP) in acute respiratory insufficiency. Chest 1975; 67:156-63.

43 Matamis D, Lemaire F, Harf A, Brun-Buisson C, Ansquer
$J C$, Atlan $G$. Total respiratory pressure-volume curves in the adult respiratory distress syndrome. Chest $1984 ; 86$ : 58-66.

44 Pesenti A, Marcolin R, Prato P, Borelli M, Riboni A, Gattinoni $L$. Mean airway pressure versus positive endexpiratory pressure during mechanical ventilation. Crit Care Med 1985; 13: 34-7.

45 Rossi A, Polese G, Brandi G, Conti G. Intrinsic positive end-expiratory pressure (PEEPi). Intensive Care Med 1995; 21: 522-36.

46 Lodato RF. Oxygen toxicity. In: Tobin MJ (Ed.). Principles and Practice of Mechanical Ventilation. New York: McGraw-Hill Inc., 1994: 837-55.

47 Lessard MR, Guérot E, Lorino H, Lemaire F, Brochard L. Effects of pressure-controlled with different I:E ratios versus volume-controlled ventilation on respiratory mechanics, gas exchange, and hemodynamics in patients with adult respiratory distress syndrome. Anesthesiology 1994; 80: 983-91.

48 Munoz J, Guerrero JE, Escalante JL, Palomino R, De La Calle $B$. Pressure-controlled ventilation versus controlled mechanical ventilation with decelerating inspiratory flow. Crit Care Med 1993; 21: 1143-8.

49 Manning HL. Peak airway pressure: why the Fuss ? Chest 1994; 105: 242-7.

50 Marini JJ, Crooke PS III, Truwit ID. Determinants and limits of pressure-preset ventilation: a mathematical model of pressure control. J Appl Physiol 1989; 67: 1081-92.

51 Rappaport SH, Shpiner R, Yoshihara G, Wright J, Chang P, Abraham $E$. Randomized, prospective trial of pressurelimited versus volume-controlled ventilation in severe respiratory failure. Crit Care Med 1994; 22: 22-32.

52 Mercat A, Graïi L, Teboul JL, Lenique F, Richard C. Cardiorespiratory effects of pressure-controlled ventilation with and without inverse ratio in the adult respiratory distress syndrome. Chest 1993; 104: 871-5.

53 Cole AGH, Weller SF, Sykes MK. Inverse ratio ventilation compared with PEEP in adult respiratory failure. Intensive Care Med 1984; 10: 227-32.

54 Tharratt RS, Allen RP, Albertson TE. Pressure controlled inverse ratio ventilation in severe adult respiratory failure. Chest 1988; 94: 755-62.

55 Lain DC, DiBenedetto R, Morris SL, Nguyen AV, Saulters $R$, Causey $D$. Pressure control inverse ratio ventilation as a method to reduce peak inspiratory pressure and provide adequate ventilation and oxygenation. Chest 1989; 95 : 1081-8.

56 Kacmarek RM, Hess D. Pressure-controlled inverse-ratio ventilation: panacea or auto-PEEP ? (Editorial) Respiratory Care 1990; 35: 945-8.

57 Mang H, Kacmarek RM, Ritz R, Wilson RS, Kimball WP. Cardiorespiratory effects of volume- and pressure-controlled ventilation at various $\mathrm{I} / \mathrm{E}$ ratios in an acute lung 
injury model. Am J Respir Crit Care Med 1995; 151 :

731-6.

58 Marini JJ, Ravenscraft SA. Mean airway pressure physiologic determinants and clinical importance - Part 2:

Clinical implications. Crit Care Med 1992; 20: 1604-16.

59 Chan $K$, Abraham $E$. Effects of inverse ratio ventilation on cardiorespiratory parameters in severe respiratory failure. Chest 1992; 102: 1556-61.

60 Shanholtz $C$, Brower $R$. Should inverse ratio ventilation be used in adult respiratory distress syndrome? Am J Respir Crit Care Med 1994; 149: 1354-8.

61 Hickling KG, Henderson SJ, Jackson R. Low mortality associated with low volume pressure limited ventilation with permissive hypercapnia in severe adult respiratory distress syndrome. Intensive Care Med 1990; 16: 372-7.

62 Kacmarek RM, Hickling KG. Permissive hypercapnia. Respir Care 1993; 38: 373-87.

63 Feihl F, Perret C. Permissive hypercapnia: how permissive should we be ? Am J Respir Crit Care Med 1994; 150: 1722-37.

64 Leatherman JW, Lari RL, Iber C, Ney AL. Tidal volume reduction in ARDS: effect on cardiac output and arterial oxygenation. Chest 1991; 99: 1227-31.

65 Kiiski R, Takala J, Kari A, Milic-Emili J. Effect of tidal volume on gas exchange and oxygen transport in the adult respiratory distress syndrome. Am Rev Respir Dis 1992; 146: 1131-5.

66 Lee PC, Helsmoortel CM, Cohn SM, Fink MP. Are low tidal volumes save? Chest 1990; 97: 425-9.

67 Marini $J J$. Inverse ratio ventilation - simply an alternative, or something more? (Editorial) Crit Care Med 1995; 23: 224-8. 


\section{New concepts in mechanical ventilation for ARDS}

Acute respiratory distress syndrome (ARDS) ${ }^{1,2}$ is associated with a high mortality of 50-70\%.,5 Although respiratory insufficiency is not the primary cause of death from ARDS, 4 the management of mechanical ventilation for these patients is problematic, as ARDS is an important risk factor of barotrauma induced by mechanical ventilation. ${ }^{6}$ The following review will discuss the management of ventilation for ARDS patients with an updated knowledge of pulmonary injury specific to ARDS.

\section{Characteristics of the pulmonary injury}

The typical lesion of ARDS is an alveolar capillary injury resulting in increased pulmonary vascular permeability. ${ }^{37.8}$ Interstitial and alveolar oedema, or high permeability oedema, results from the injury to the alveolar capillary membrane. This alveolar capillary involvement is the result of either a direct (e.g., aspiration) or indirect insult (e.g., septic shock, severe trauma, multiple transfusions) ${ }^{3-5}$ Although the increase in vascular pulmonary permeability appears to affect the lungs in a diffuse fashion, the alveolar oedema is not evenly distributed. ${ }^{8}$ Indeed, even if the alveolar infiltrates appear evenly spread on a chest $x$-ray, CT-scan studies show that the infiltrate distribution is not homogeneous. ${ }^{9-13}$ The pulmonary injury is most evident in the dependant zones (dorsal for a supine patient) while the non-dependant zones (ventral for the prone patient) remain intact. ${ }^{14}$ In ARDS, the lungs are divided according to a three zone model. ${ }^{12}$ The pulmonary non dependant zone receives all the ventilation and is often hyperventilated in relation to the number of its alveolar units. The second intermediate zone consists of unventilated alveolar units that can be recruited and made available for ventilation by changes in the settings of the mechanical ventilator. ${ }^{13}$ Finally, a third pulmonary zone, the most likely affected and usually the most dependant, is impossible to ventilate. ${ }^{11-14}$ In ARDS, the aerated pulmonary zone, that is available for ventilation, is considerably diminished and may represent as little as $20-30 \%$ of the total lung capacity. ${ }^{11,12,15}$ It is the concept of "baby lungs" proposed by Gattinoni et al. ${ }^{12}$ Also, in ARDS, there is a substantial decrease in pulmonary static compliance. Poor static compliance is mainly the result of de- creased volume in the the ventilated lung. ${ }^{11}$ Pulmonary elasticity is best measured with the specific compliance, i.e., static compliancewith a corrcetion for the pulmonary volume available for ventilation. ${ }^{16}$ Specific compliance is very little changed in ARDS, implying that the elasticity of the ventilated pulmonary zones is almost normal..$^{11,15,17}$ These zones receive the whole of the ventilation and behave mechanically like normal lung tissue. This functional amputation of the pulmonary volume represents the primary pathological change and explains why the ventilatory approach to the ARDS patient must be modified when compared with patients with normal lungs.

\section{Injuries associated with mechanical ventilation}

It is well recognised that mechanical ventilation is associated with complications induced by barotrauma (pneumothorax, pneumomediastinum, interstitial emphysema) ${ }^{6,18}$ However, in the past few years, it has been shown in animal studies that mechanical ventilation using high tidal volumes and pressures could rapidly alter alveolar capillary permeability and cause the type of diffuse damage seen in ARDS. ${ }^{18-23}$ Furthermore, the major determinant of alveolar injury is overdistension of the alveoli, implying that the alveolar volume and not the high pressures used for ventilation are responsible. ${ }^{20,24}$ It has been suggested that the term "volotrauma" might be more appropriate than barotrauma for the description of pulmonary injuries associated with ventilation. ${ }^{25}$ Concerning ventilation of normal lungs, the clinical importance of these studies is unclear. However, with a marked decrease in pulmonary capacity available for ventilation in ARDS, high volume ventilation is likely to increase the risk of alveolar injury. The injuries associated with mechanical ventilation are the cause of the complications and aggravate respiratory insufficiency.

\section{Objectives of ventilation in ARDS (Table)}

1 to provide adequate gas exchanges;

2 to minimise the risk of pulmonary injury associated with mechanical ventilation by performing the appropriate ventilator adjustments for ARDS.

During mechanical ventilation tidal volumes of 10-15 
TABLE Principles of management of ARDS patients

\begin{tabular}{lll}
\hline Ventilation mode & Volume control & Assist-control, SIMV \\
\hline Tidal volume & $5-7 \mathrm{ml} \cdot \mathrm{kg}^{-1}$ & Must be decreased if $\mathrm{P}_{\text {plat }}>35 \mathrm{cmH}_{2} \mathrm{O}$ at the upper inflection point \\
PEEP & $5-15 \mathrm{cmH}_{2} \mathrm{O}$ & Best determined with the lower inflexion point on the P/V curve \\
Respiratory rate & $\leq 20-25 \mathrm{~min}^{-1}$ & Avoid auto-PEEP \\
$\mathrm{FIO}_{2}$ & $\leq 0.50$ & Increase to $\mathrm{SaO}_{2} \geq 90 \%$ (if $\mathrm{PEEP} \geq$ lower inflection point or $\geq 15 \mathrm{cmH}_{2} \mathrm{O}$ ) \\
$\mathrm{I}: \mathrm{E}$ ratio & $\leq 1.1$ & Avoid auto-PEEP \\
$\mathrm{P}_{\text {plat }}$ & $\leq 35 \mathrm{cmH}_{2} \mathrm{O}$ & Best determined with the upper inflexion point on the P/V curve \\
\hline
\end{tabular}

$\mathrm{P}_{\mathrm{plat}}=$ Inspiratory plateau pressure; $\mathrm{P} / \mathrm{V}$ curve = pressure/volume curve according to the pressure/volume curve of the respiratory system.

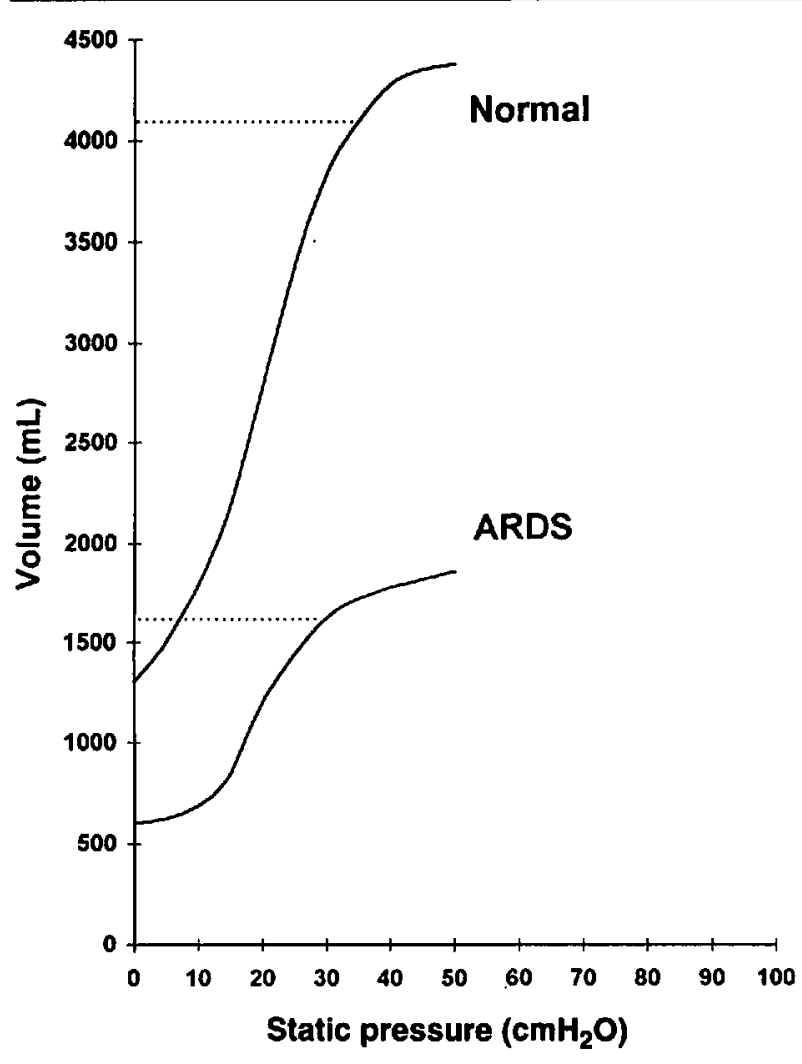

FIGURE 1 Pressure-volume curves of mechanically ventilated patients without ARDS (Normal) and with ARDS. Both curves have the same pattern except for the reduced FRC and the upper plateau (upper inflexion point) occurring in the ARDS patient at a lower volume. This Figure shows the functional amputation of the pulmonary volume typical to ARDS.

$\mathrm{ml} \cdot \mathrm{kg}^{-1}$ are often used. ${ }^{26,27}$ Although, these are twice the spontaneous tidal volume, these large volumes probably do not represent a high risk for patients with healthy lungs and a normal pulmonary capacity. However, in severe ARDS, the pulmonary capacity is only $20-30 \%$ of normal and must receive the entire ventilation. ${ }^{11,12}$ This implies that each of the ventilated pulmonary units must accept a volume six to eight times its normal alveolar tidal volume. In conformity with the

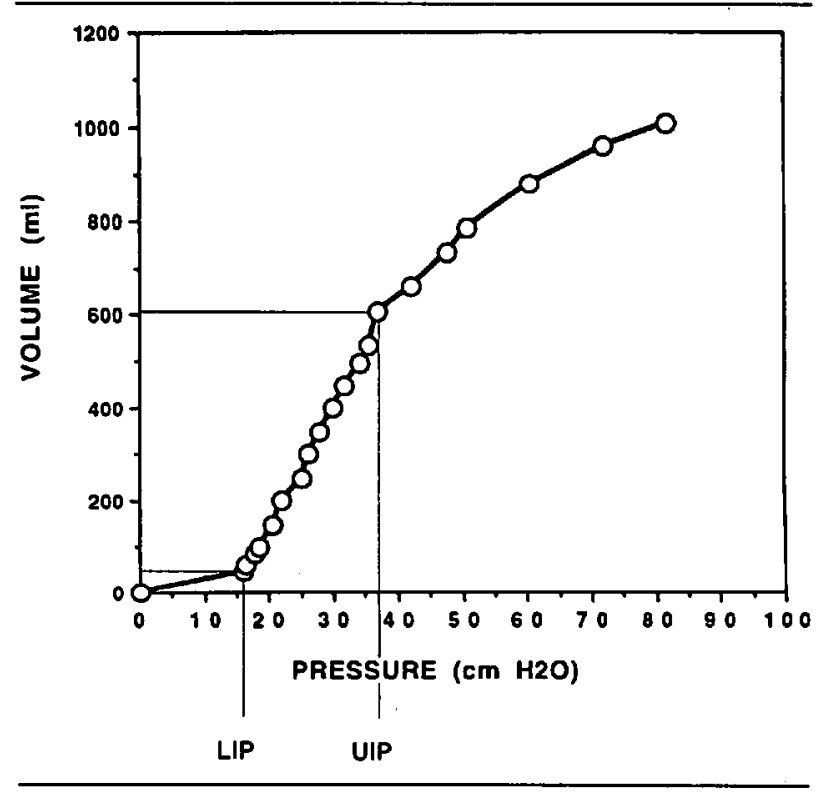

FIGURE 2 Pressure-volume curve of an ARDS patient. The upper (UIP) and lower (LIP) inflexion points are easily identified. The UIP is set at $36 \mathrm{~cm} \mathrm{H}_{2} \mathrm{O}$ corresponding to a volume larger than FRC by $600 \mathrm{ml}$. The LIP corresponds to the point where the respiratory compliance improves abruptly. (With permission of Roupie $E$ and Brochard L. Créteil, France)

"baby lung" pattern of ARDS, ${ }^{1}$ this corresponds to ventilating a $15 \mathrm{~kg}$ child with a tidal volume of $600-800 \mathrm{ml}$. In animals, alveolar overdistension of this magnitude rapidly induces alveolar injury and rupture of the alveolar capillary membrane with pulmonary oedema. ${ }^{19-23}$

As in the normal patient, in ARDS the pressure/volume relationship of the respiratory system is sigmoid in shape with a high plateau indicating that the total pulmonary capacity has been reached ${ }^{28}$ (Figure 1). However, in the course of ARDS, this flattening of the curve occurs at a lower volume, reflecting the functional amputation due to ARDS. ${ }^{28,29}$ The point where this flattening starts is called the upper inflexion point (Figure 2). Roupie et al. have shown that ventilation with a tidal volume of $10 \mathrm{ml} \cdot \mathrm{kg}^{-1}$ exceeds the pressure and the volume corresponding to the superior inflexion point in 


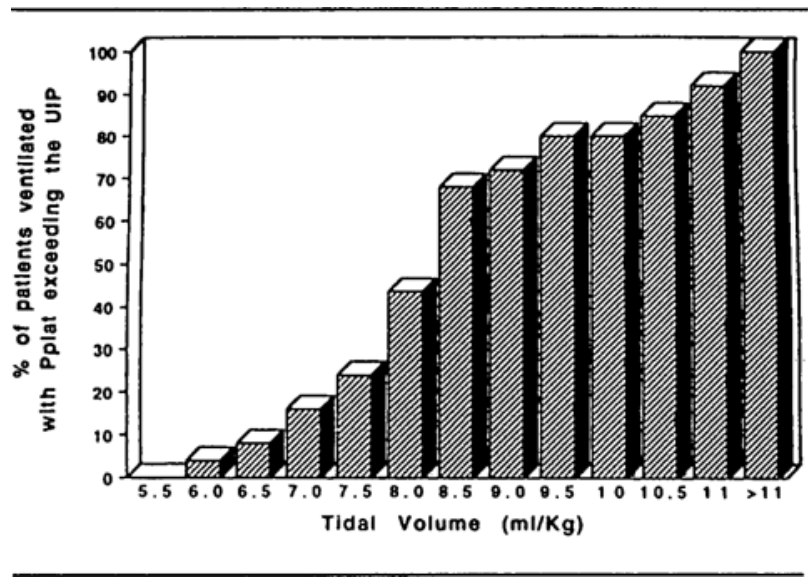

FIGURE 3 Percentage of patients with ARDS whose the inspiratory pressure plateau exceeded the UIP of the pressure-volume curve relative to tidal volume (with a PEEP of $10 \pm 3 \mathrm{~cm} \mathrm{H}_{2} \mathrm{O}$ ). When tidal volume $\geq 10 \mathrm{ml} \cdot \mathrm{kg}^{-1}$, LIP is exceeded in $80 \%$ of patients. (with permission of Roupie et al.).

more than $80 \%$ of ARDS patients (Figure 3). ${ }^{29}$ These ventilatory volumes may thus induce alveolar overdistension and associated "barovolotrauma." It is recommended that the tidal volume be limited to $5-7 \mathrm{ml} \cdot \mathrm{kg}^{-1}$ in patients with ARDS. ${ }^{30-33}$ Ideally, the tidal volume setting should be determined by the measured pressure/volume curve of the patient. ${ }^{29-34}$ If this is not possible, the alveolar pressure may be monitored by assessment of the inspiratory plateau pressure. Tidal volume must be adjusted to keep the pressure of the inspiratory plateau $<35 \mathrm{cmH}_{2} \mathrm{O}$. However, this value is arbitrary, does not account for the wall compliance and frequently exceeds the upper inflexion point of the pressure/volume curve. ${ }^{29}$ Analysis of the dynamic pressure curve of ventilation on a graphic recorder or on the screen of the respirator monitor has been proposed by others. Using constant inspiratory flow, acceleration of the end-expiratory pressure (i.e., a concavity at the top of the curve) suggests that the maximum alveolar capacity has been reached. ${ }^{36}$

\section{PEEP}

It has been clearly established that prophylactic PEEP does not prevent ARDS. ${ }^{37}$ Added PEEP recruits more alveoli and keeps them open during the whole respiratory cycle and thus improves arterial oxygenation. PEEP increases the functional residual capacity which is greatly decreased in ARDS. ${ }^{15}$ While maintaining the ventilated alveoli open, PEEP not only increases oxygenation, but also decreases the alveolar duct closure and reopening cycle occurring with each respiration. Studies on living animals showed that the pressure required to re-open those small airway repeatedly causes lung damage. 22.38 This can be reduced by using a PEEP level capable of increasing end expiratory pulmonary volume and of maintening the patency of the small airways during the whole respiratory cycle. ${ }^{38}$ PEEP has two benefits during ARDS: it improves arterial oxygenation and decreases the pulmonary injury associated with ventilation. However, PEEP also has adverse side effects. The decrease in cardiac output is well known and sometimes warrants invasive monitoring. In addition, the alveolar injury produced by PEEP may also cause alveolar damage identical to that of large ventilatory volumes. ${ }^{23}$ The PEEP effect is not limited to the recruitable lung areas. ${ }^{39}$ Even if PEEP encourages recruitment and patency of new alveolar units, it also increases the end expiratory volume of pulmonary areas that are adequately ventilated. This increase in volume added to the tidal volume effect can lead to overdistension of some alveolar units and increase the risk of alveolar injury if the end-inspiratory volume (i.e., tidal volume plus volume induced by PEEP) come any closer to the maximum alveolar capacity (i.e., the upper inflection point). ${ }^{23,29,40}$

The optimal level of PEEP is often determined by assessment of oxygen transport. ${ }^{41}$ However, this may lead to the use of very high levels of PEEP ${ }^{42}$ with an added risk of alveolar overdistension. Measuring the respiratory pressure-volume curve may be of some help in adjusting PEEP (Figure 2). The lower part of the curve is usually distinguished by a change in the slope of the curve, the lower inflexion point. This point correspond to the PEEP level required for maximal alveolar recruitment. ${ }^{11,15,38,43,44}$ This optimal PEEP level is considered as the minimal level that should be used to keep the small airways open at end expiration. At a higher level, PEEP will probably increase the volume of alveoli already ventilated. ${ }^{11,15,44}$ Planned PEEP adjustments must aim at improving arterial oxygen and transport, keeping open a maximal number of alveolar units and avoiding alveolar overdistension. If it is impossible to measure the pressure/volume curve, it is best to increase PEEP progressively while monitoring both the changes in arterial oxygenation and that of the inspiratory plateau pressure. In practice, the optimal PEEP level is in the range of $8-15 \mathrm{cmH}_{2} \mathrm{O}$ (sometimes higher if the wall compliance is decreased). ${ }^{15,41}$ It is important to remember that any increase in PEEP may require a decrease of the tidal volume to sustain the end-expiratory volume (maximal alveolar volume) and to maintain the plateau pressure below the upper inflexion point (or $<35 \mathrm{cmH}_{2} \mathrm{O}$ ). ${ }^{23,29,40}$

\section{Respiratory rate}

During conventional mechanical ventilation, the rate is set to $10-12 \mathrm{~min}^{-1}$ and this is usually adequate to provide normal alveolar ventilation and $\mathrm{PaCO}_{2}$ because of 
the large volumes used. If tidal volume is reduced, it is expected that the rate would need to be increased. However, it is difficult and hazardous to increase rates $>20-25 \mathrm{~min}^{-1}$. Indeed, it would lead to an excessive decrease of respiratory cycle duration and therefore of expiratory time. Incomplete expiration will cause pulmonary overinflation with auto-PEEP. ${ }^{45}$ The increased end-expiratory volume together with the volume induced by respirator PEEP, results in considerable pulmonary hyperinflation and increases the risk of alveolar overdistension.

\section{$\mathrm{FIO}_{2}$}

The aim is to provide adequate oxygen saturation $\left(\mathrm{SaO}_{2}\right.$ $\geq 90 \%$ ) with a non toxic $\mathrm{FIO}_{2}(\leq 0.60) .{ }^{31}$ In very severe ARDS where a high oxygen concentration is required, one must choose between two evils: the risk of alveolar overdistension associated with an increased PEEP or the risk of oxygen toxicity. ${ }^{46}$ In such a situation, it might be preferable to face the risk of barovolotrauma than the risk of oxygen toxicity.

\section{Controlled volume or pressure controlled ventilation}

Volume controlled (CV) ventilation is associated with high peak pressures. To restrain the pressure and perhaps decrease barotrauma, pressure controlled (PC) ventilation has been proposed as an alternative. If both modes are compared in the same ventilatory setting (tidal volume, rate, PEEP), the peak pressure is reduced in PC mode. However, the level of the inspiratory plateau pressure is similar. ${ }^{47,48}$ Any change in peak pressure induced by manipulating the inspiratory flow pattern or by reducing inspiratory flow will not alter the risk of pulmonary injury if it is not combined with a decrease of the alveolar pressure (inspiratory plateau pressure), tidal volume or PEEP. ${ }^{23,49}$ The risk of pulmonary injury is depends on the magnitude of the alveolar distension volume and not on the ventilatory mode delivering that volume. ${ }^{20,23}$ One disadvantage of $\mathrm{PC}$ is that tidal volume and therefore minute-ventilation are not guaranteed; much closer monitoring of both ventilatory variables and gas exchanges is required. ${ }^{50}$ While $P C$ ventilation may adequately ensure gas exchange in ARDS patients, ${ }^{51}$ there are no studies or theoretical evidence suggesting that $\mathrm{PC}$ is better than $\mathrm{CV}$ ventilation. ${ }^{37,47,48}$

\section{Inverse ratio ventilation}

Inverse ratio ventilation (IRV) can be used in the volume control (IRV-VC) or PC control mode (IRV-PC). ${ }^{35}$ The increased duration of inspiration allows a reduction in inspiratory flow and thus in the peak ventilatory pressure. However, as already discussed, one must not expect to be able to reduce the risk of barovolotrauma if the pulmonary volume and inspiratory plateau pressure remain unchanged. The increased inspiratory time implies an equivalent decrease of the expiratory time associated with incomplete lung expansion. This results in an increase of end-expiratory volume with autoPEEP. ${ }^{35,45,50,52,53}$ The auto-PEEP increases very rapidly when the expiratory time is less than one second (i.e., I:E ratio of 2:1 with a respiratory rate of 20 per $\mathrm{min}$ ). ${ }^{35,50}$ Auto-PEEP induced by IRV adds to the PEEP generated in the respirator circuit and, consequently, very high levels of end-expiratory alveolar pressure (Total PEEP) and hyperinflation may occur. Some instances of improved arterial oxygenation with IRV were initially reported ${ }^{54,55}$ However, the PEEP level was not measured and accounted for. ${ }^{56}$ If IRV is compared with conventional mechanical ventilation in identical settings for tidal volume, rate and total PEEP, no improvement of arterial oxygenation is observed. ${ }^{47,52,58,59}$ The use of IRV requires close respiratory monitoring (tidal volume, minute-ventilation, total PEEP) and invasive cardiovascular monitoring. In summary, even if this ventilatory mode is proposed, there is no evidence or controlled studies to suggest the superiority of IRV over conventional ventilation. Therefore, IRV does not appear to be appropriate for the treatment of ARDS.

\section{Permissive hypercapnia}

In severe ARDS, the alveolar ventilation provided by the use of limited tidal volumes $\left(5-7 \mathrm{ml} \cdot \mathrm{kg}^{-1}\right)$ and also limited respiratory rate $\left(\leq 25 \mathrm{~min}^{-1}\right)$ will be adequate to fulfil ventilatory needs and maintain normal $\mathrm{PaCO}_{2}$. This condition is referred to as controlled hypoventilation or permissive hypercapnia. ${ }^{36,61,62}$ Generally, $\mathrm{PaCO}_{2}$ rises to $60-80 \mathrm{mmHg}$ and the initial $\mathrm{pH}$ decreases to around 7.25. This degree of acidosis is usually well tolerated and there is no need to intervene. $29,33,61-63$ Intracellular $\mathrm{pH}$ returns to normal within a few hours and extracellular $\mathrm{pH}$ returns to near normal levels within two or three days due to normal metabolic compensation. ${ }^{63}$ Under certain conditions, more severe hypercapnia and acidosis may occur $\left(\mathrm{PaCO}_{2}>110 \mathrm{mmHg}, \mathrm{pH}\right.$ 7.0) ${ }^{32}$ Some clinicians do not correct this acidaemia while others suggest that metabolic compensation should be accelerated with the use of a slow infusion of sodium bicarbonate. ${ }^{64-66}$ Acute and chronic hypercapnia appears to be well tolerated by animals and humans as long as adequate oxygenation is provided.$^{63}$ Numerous studies have shown that low volume ventilation is well tolerated with respect to arterial oxygen and haemodynamic variables. ${ }^{64-66}$ However, deep sedation and sometimes, muscular relaxation is required for the patient's comfort. Some contraindication need to be considered. 
Head trauma or other causes of increased intracranial pressure are absolute contraindications to increases in $\mathrm{PaCO}_{2}$. Cardiovascular instability is also a contraindication. Associated metabolic acidosis should be corrected to avoid excessive acidaemia. Retrospective and prospective studies suggest that the mortality rate associated with limited ventilation and permissive hypercapnia has declined. ${ }^{32,61}$ Prospective randomised studies that could confirm these encouraging results are presently performed.

\section{Conclusion}

With regard to respiratory physiology, ARDS is characterised by functional amputation of the pulmonary volume. In severe cases, as few as $20-30 \%$ of alveolar units are open for ventilation. High volume conventional ventilation may cause additional injury to the ARDS lungs. The ventilatory approach must be modified and adapted to the physiological characteristics of ARDS lungs. Sufficient PEEP for maximal recruitment of alveoli must be provided while avoiding alveolar overdistension associated with high levels of PEEP. Tidal volume must be reduced $\left(5-7 \mathrm{ml} \cdot \mathrm{kg}^{-1}\right)$ to limit the alveolar end inspiratory volume and therefore decrease the risk of lung injury. This type of ventilation will often be adequate to fill the respiratory needs of the patient. This ventilation strategy is called "permissive ventilation." Finally, there is no theoretical evidence or controlled studies to prove that IRV or PC ventilation are better to VC ventilation: "The skilful management of ARDS is a complex undertaking that requires mastery of the fundamental principles of lung protection and gas exchange efficiency,... As always, the best approach to such problems is a well-reasoned strategy firmly grounded in applied physiology." (From Marini JJ. ${ }^{67}$ )

\section{References}

(See page R47) 